\title{
ROBUST BLIND SCATTERER SEPARATION IN MULTIBASELINE INSAR
}

\author{
Yuanyuan Wang $^{1}$, Xiao Xiang Zhu ${ }^{1,2}$
}

\section{Signal Processing in Earth Observation, Technical University of Munich 2. Remote Sensing Technology Institute, German Aerospace Center}

\begin{abstract}
The side-looking imaging geometry of synthetic aperture radar (SAR) causes inevitable layover in SAR images. Separating the contributions from different scatterers has been the fundamental for many applications. It is typically solved by explicit inversion of the SAR imaging model to retrieve the scattering profile along the mixed dimension (elevation), which is otherwise known as SAR tomography. This paper proposed a robust blind scatterer separation method to demix the layovered scatterers, avoiding the computationally expensive tomographic inversion. We demonstrate that the state-of-the-art principle component decomposition-based methods are heavily influenced by the nonergodicity of the selected samples, especially in urban area, such as point scatterers appearing often on facades. The proposed method is shown to be more robust than the state-of-the-art. Real data example shows that the proposed method outperforms the state-of-the-art by a factor of three in terms of the accuracy of the retrieved phase.
\end{abstract}

Index Terms - blind source separation, tomographic inversion, multi-baseline, robust estimation, covariance matrix, InSAR, SAR

\section{INTRODUCTION}

The side-looking imaging geometry of synthetic aperture radar (SAR) causes inevitable layover in SAR images, especially in mountainous and urban areas. The layover is usually separated by SAR tomographic inversion (TomoSAR) which is the strict SAR 3-D SAR imaging. Because of the ability of layover separation, TomoSAR is the most competent multibasline InSAR method for the monitoring of urban areas. However, such inversion is much more computationally expensive than methods based on single-scatterer model, such as persistent scatterer interferometry (PSI), because it requires the inversion of a much larger equation system plus maxima detection in the retrieved reflectivity profile. This hinders TomoSAR to be widely applied for deformation monitoring.

Recently, [1] proposed a principle component analysis (PCA)-based method to blindly separate the phase of multiple scatterers without explicit performing the tomographic inversion. In a more general context, this is known as blind source separation in signal processing. It showed that the dominant eigenvectors of the sample covariance matrix can be regarded as the steering vectors of individual scatterers layovered in a pixel. Reasonable results were also demonstrated.

In this paper, we show that such correspondence between eigenvectors and scatterers is only mildly fulfilled under certain conditions. In addition, such method is based on the second order statistics of Gaussian scatterers, and hence its performance degrades when dealing with heterogeneous scattering mechanism in urban areas. This paper proposes a robust blind scatterer separation method in urban areas using robust covariance matrix estimation techniques.

\section{BACKGROUND}

\subsection{Mixing model}

\section{Fully coherent model (semi-PS)}

In urban area, the reflectivity profile can be often assumed to be fully coherent over the multiple acquisitions. Under such assumption, the discrete SAR imaging model can be expressed as follows.

$$
\mathbf{g}=\mathbf{R} \boldsymbol{\gamma}+\boldsymbol{\varepsilon}
$$

where $\mathbf{g} \in \mathbb{C}^{N}$ is the multibaseline SAR measurements, $\mathbf{R} \in \mathbb{C}^{N \times L}$ is the so called steering matrix which is basically a discrete Fourier transform matrix, $\gamma \in \mathbb{C}^{L}$ is the reflectivity profile along the elevation direction, $\boldsymbol{\varepsilon} \in \mathbb{C}^{N}$ is the identically and independently distributed (i.i.d.) noise vector, and $N, L$ are the number of images and the discretization level in the elevation direction, respectively. In urban area, the profile $\gamma$ is usually consisted of a few dominant peaks. For a $K$-scatterer profile, the imaging model can be simplified to

$$
\mathbf{g}=\left[\begin{array}{llll}
\mathbf{r}_{1} & \mathbf{r}_{2} & \cdots & \mathbf{r}_{K}
\end{array}\right]\left[\begin{array}{c}
\gamma_{1} \\
\gamma_{2} \\
\vdots \\
\gamma_{K}
\end{array}\right]+\boldsymbol{\varepsilon},
$$

where $\mathbf{r}_{k} \in \mathbb{C}^{N}$ is the steering vector of the $k$ th scatterer. The covariance matrix of the observations $\mathbf{g}$ is then

$$
\mathbf{C}=\mathrm{E}\left\{\mathbf{g g}^{H}\right\}=\mathbf{R} \mathrm{E}\left\{\boldsymbol{\gamma} \boldsymbol{\gamma}^{H}\right\} \mathbf{R}^{H}+\mathrm{E}\left\{\boldsymbol{\varepsilon} \boldsymbol{\varepsilon}^{H}\right\}
$$


where $(\bullet)^{H}$ is the conjugate transpose operator. The profile $\gamma$ can be assumed to be uncorrelated, which leads $\mathrm{E}\left\{\gamma \gamma^{H}\right\}$ to a diagonal matrix with the expected intensity of individual scatterers. The observation covariance matrix can be expressed as

$$
\mathbf{C}=\sum_{k=1}^{K} \sigma_{k}^{2} \mathbf{r}_{k} \mathbf{r}_{k}^{H}+\sigma_{\varepsilon}^{2} \mathbf{I}
$$

where $\sigma_{k}^{2}$ is the expected intensity of the $k$ th scatterer, and $\sigma_{\varepsilon}^{2} \mathbf{I}$ accounts for the covariance matrix of the noise. Without losing generality, we can assume the steering vectors $\mathbf{r}_{k}$ are all normalized.

Such scattering model resembles the layover of PSs. However, it is worth mention that the intensities across different looks are not assumed to be deterministic. They are assumed to be the realization of complex circular Gaussian random variables, i.e. Gamma-distribution with two degree of freedom. Such semi-deterministic model is more practical to describe the PS behavior in real SAR images. This model is known to the radar jargon as Swerling II model [2].

\section{Decorrelating model (DS)}

For a more general purpose, the decorrelation of the reflectivity profile $\boldsymbol{\gamma}$ due to geometric or temporal decorrelation needs to be considered. For a $K$-scatterers mixture, the imaging model can be formulated as follows [1], [3]:

$$
\mathbf{C}=\sum_{k=1}^{K} \mathbf{r}_{k} \mathbf{r}_{k}^{H} \odot \mathbf{C}_{k}+\sigma_{\varepsilon}^{2} \mathbf{I},
$$

where $\mathbf{C}_{k} \in \mathbb{R}^{N \times N}$ is its real-valued covariance matrix containing the intensity of the $k$ th scatterer and its decorrelation matrix. The symbol $\odot$ denotes an elementwise product. The fully-coherent model is a special case of the decorrelating model where $\mathbf{C}_{k}$ degenerates to constant matrices. Despite model (5) taking into account the decorrelation being more general, it requires additional modeling of the decorrelation covariance tensor, and thus the estimation of additional parameters. Therefore, this paper adopts the fully-coherent model in equation (4), which can be often fulfilled in urban areas.

\subsection{PCA-based methods and limitations}

PCA-based method [1] retrieves the steering vectors of individual scatterers by performing eigenvalue decomposition on the covariance matrix:

$$
\mathbf{C}=\mathbf{V D V}^{H}
$$

where $\mathbf{V}$ is the eigenvectors, and $\mathbf{D}$ is the diagonal matrix of the eigenvalues. According to [1], the individual eigenvectors correspond to the signal directions, i.e. steering vectors, of individual scatterers. And hence, the steering vectors can be estimated by

$$
\hat{\mathbf{r}}_{i}=\left\|\mathbf{v}_{i}\right\|_{2}^{-1} \mathbf{v}_{i}
$$

where $\mathbf{v}_{i}$ is the columns of $\mathbf{V}$. This strictly assumes the steering vectors of different scatterers are orthogonal, which can only be mildly fulfilled when the elevation between scatterers are large.

\section{Limitations}

The limitation of [1] is two-fold. On one hand, it is performed on sample covariance matrix whose estimation is a major challenge in urban areas, due to the heterogeneous scattering mechanism among the neighboring samples. It is also nearly impossible to find sufficient samples whose scattering centers of individual scatterers are identical to the corresponding ones in each sample respectively.

On the other hand, PCA assumes a linear combination of orthogonal basis. Therefore, it is not able to fully recover nonorthogonal basis. In reality, the steering vectors of difference scatterers are seldom completely orthogonal. In addition, the directions of the sources are indeterminable when the variances of the individual Gaussian sources are identical. This can be demonstrated by a 2-D Gaussian mixture shown in Figure 1, where the two subfigures show 2-D mixtures of nonorthogonal and orthogonal Gaussians of identical variance. The solid arrows are the true direction of the mixed source, and the dashed arrows are the directions extracted by PCA. The length of the arrows is the three standard deviation of the (estimated) Gaussian distribution. The subfigure (a) shows that nonorthogonal mixture of Gaussian sources cannot be separated by PCA, while subfigure (b) shows that orthogonal mixture of Gaussian sources with the same variance can also not be unmixed by PCA, because PCA is rotation invariant at identical variance of individual Gaussian sources.

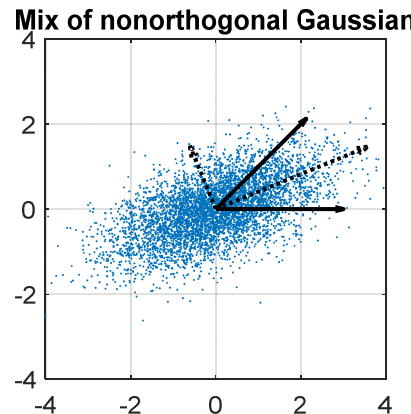

(a)

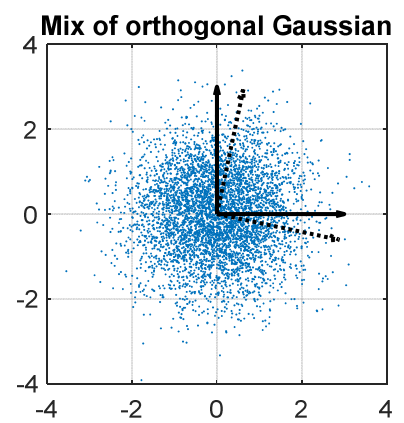

(b)
Figure 1. 2-D mixture of (a) nonorthogonal and (b) orthogonal Gaussians of identical variance (set to 1 ). The solid arrows are the true direction of the mixed source, and the dashed arrows are the directions extracted by PCA. The length of the arrows is the three standard deviation of the Gaussian distribution. The subfigure (a) shows that nonorthogonal mixture of Gaussian sources cannot be separated by PCA, while subfigure (b) shows that orthogonal mixture of Gaussian sources with the same variance can also not be unmixed by PCA. 
These limitations cause systematic phase bias in the estimated steering vectors. The bias increases as the intensity or the elevation distance of the scatterers get closer.

\section{ROBUST BLIND SCATTERER SEPARATION}

To improve the existing method, we make use of robustly estimated covariance matrix in the blind scatterer separation.

\subsection{Robust Covariance matrix estimation}

The maximum likelihood estimator (MLE) for the covariance matrix under the assumption of Gaussian scatterers is simply the sample covariance matrix:

$$
\hat{\mathbf{C}}_{M L E}=\frac{1}{M} \sum_{m=1}^{M} \mathbf{g}_{m} \mathbf{g}_{m}^{H}
$$

where $\mathbf{g}_{m}$ is the $m$ th sample, and $M$ is the total number of samples. As shown in [4], the MLE is not robust against non-Gaussian scatterers. It can heavily overestimate covariance matrix, especially in urban area. This is usually caused by the bright point scatterers which are selected in the samples for the covariance estimation.

A general form of robust covariance estimator can be expressed by a weighted sample covariance matrix as follows [4], [5]:

$$
\hat{\mathbf{C}}=\frac{1}{M} \sum_{m=1}^{M} w\left(\mathbf{g}_{m}^{H} \mathbf{C}^{-1} \mathbf{g}_{m}\right) \mathbf{g}_{m} \mathbf{g}_{m}^{H}
$$

where $w(\bullet)$ is a real-valued weighting function. The weight depends on the negative $\log$ likelihood $\mathbf{g}_{m}^{H} \mathbf{C}^{-1} \mathbf{g}_{m}$. It downweights highly deviating samples whose likelihood is low, which greatly depends on the intensities of $\mathbf{g}_{m}$. Equation (9) can be solved by an iteratively reweighted approach [5], [6]:

$$
\hat{\mathbf{C}}_{n+1}=\frac{1}{M} \sum_{m=1}^{M} w\left(\mathbf{g}_{m}^{H} \hat{\mathbf{C}}_{n}^{-1} \mathbf{g}_{m}\right) \mathbf{g}_{m} \mathbf{g}_{m}^{H}
$$

where $n$ denotes the iteration index.

One robust weighting function is $w=\left(\mathbf{g}_{m}^{H} \mathbf{g}_{m}\right)^{-1}$. The corresponding covariance matrix estimator is

$$
\hat{\mathbf{C}}_{S C M}=\frac{1}{M} \sum_{m=1}^{M}\left\|\mathbf{g}_{m}\right\|^{-2} \mathbf{g}_{m} \mathbf{g}_{m}^{H} .
$$

This is also known as the sign covariance matrix (SCM) [7], [8], where only the direction of each multivariate sample is considered. The real covariance is lost as a result. However, the interferometric phase is preserved in the estimation. SCM does not require iteration and is robust against outliers.

\section{EXPERIMENTS}

We compared the proposed method and the state-of-the-art on a test building (Bellagio Hotel, Las Vegas). Figure 2 shows the optical and the SAR amplitude image of the building. The yellow arrows on the images indicate the line of sight of the sensor, which implies that the façade is layovered with the uneven ground in front of it. Seeing from the optical image, the façade is fairly vertical, but the ground consists of various different objects, e.g. small structure, swimming pool, etc. The whole data stack comprises 30 TerraSAR-X high resolution spotlight images.

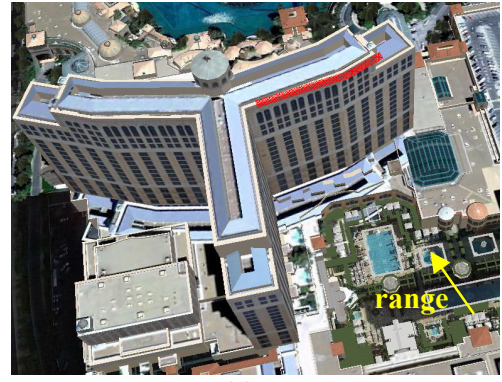

(a)

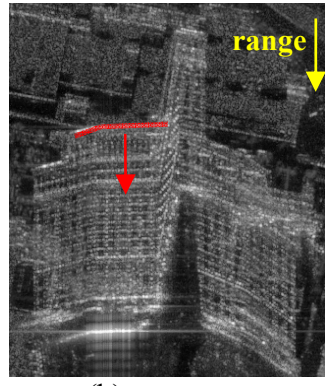

(b)
Figure 2. (a) the Google optical image of the test building, and (b) the SAR amplitude image.

To select samples for estimating covariance matrix, we did not follow the approach employed in [1] which is a statistical test based on amplitude. The reason is that such statistical test does not consider the phase center of the samples. Instead, an iso-height template is manually drawn in the SAR image, which are shown as red curves in Figure 2. The covariance matrices can be estimated in a sliding window manner. Such iso-height template is the ideal sample selection window for layovered flat façade and flat ground. Despite the fact that the ground layer in the test area is not even, such window can at least make sure the phase centers of the façade scatterers in each sample are nearly identical.

The template was slid from near-range to far-range (top down). By performing the proposed method, we extract the two most dominant eigenvectors at each template position. They are regarded as the phase centers of the façade and the ground (or roof) scatterers. Afterwards, the height of each scatterer can be individually retrieved using single-scattererbased approach, e.g. PSI.

Figure 3 shows the height retrieved from the proposed method (right) and the state-of-the-art (left). The samples used for the estimation of the covariance matrices are identical. The figure shows the estimated heights of the two layers w.r.t. to the template position. Ideally, one should expect some small structures on the ground and a fairly straight line representing the façade. However, there are many outliers in the estimated height using the state-of-theart method. The façade points also have certain systematic bias appearing as small undulation. Therefore, the source 
separation is not ideal, despite the samples have been optimally selected in the best possible manner.

In contrast, the façade height shows a much more linear structure in the result from the proposed method. The height estimates of the second layer are also much more consistent and robust. More detailed ground structures have been revealed. In particularly, the proposed method also resolved the layover between the roof and the façade, which is marked by the black ellipse in Figure 3(b). By assuming the façade is a straight line, the proposed method outperforms the state-of-the-art by a factor of threes in terms of the accuracy of the height estimates.

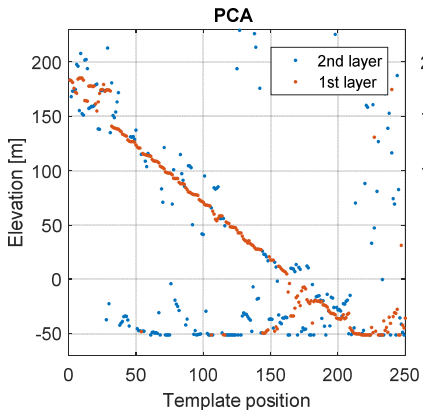

(a)

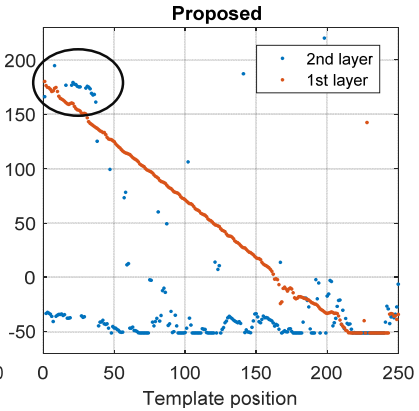

(b)
Figure 3. The height estimated from the two most dominant eigenvectors from (a) the sample covariance matrix, and (b) the sign covariance matrix. The black ellipse marks the layover region between the façade and the roof. In the result of the proposed method, the façade height shows a much more linear structure. The height estimates of the second layer are also much more consistent and robust. More detailed ground structures have been revealed. In particularly, the proposed method also resolved the layover between the roof and the façade.

\section{CONCLUSION AND OUTLOOK}

We demonstrated a robust method to blindly separate multiple scatterers layover in a SAR image pixel. It was shown that the state-of-the-art method can obtain reasonable result only under certain conditions, i.e. large elevation and intensity difference between the scatterers. Using real data, it was shown that the proposed method outperforms the state-of-the-art by a factor of three in terms of the accuracy of the interferometric phase estimates, and in turn the accuracy of the height derived from the phase. The experiment also tells that a robust covariance estimator is necessary in urban area, even if the samples are optimally selected. The large dynamic range and the complex scattering mechanism of the scatterers often cannot guarantee a reliable estimate of the covariance matrix.

Nevertheless, both methods still strictly assume orthogonality between the steering vectors of different scatterers. This implies that systematic phase bias is inevitable in the estimated steering vectors. Therefore, we are currently developing algorithms without this assumption.

\section{ACKNOWLEDGEMENT}

The authors would like to thank Prof. R. Bamler for valuable discussion.

\section{REFERENCES}

[1] G. Fornaro, S. Verde, D. Reale, and A. Pauciullo, "CAESAR: An Approach Based on Covariance Matrix Decomposition to Improve Multibaseline-

Multitemporal Interferometric SAR Processing," IEEE Trans. Geosci. Remote Sens., vol. 53, no. 4, pp. 20502065, Apr. 2015.

[2] M. A. Richards, J. A. Scheer, and W. A. Holm, Eds., Principles of Modern Radar: Basic Principles. Raleigh, NC: SciTech Publishing, 2010.

[3] F. De Zan, M. Zonno, and P. Lopez-Dekker, "Phase Inconsistencies and Multiple Scattering in SAR Interferometry," IEEE Trans. Geosci. Remote Sens., vol. 53, no. 12, pp. 6608-6616, Dec. 2015.

[4] Y. Wang and X. X. Zhu, "Robust Estimators for Multipass SAR Interferometry," IEEE Trans. Geosci. Remote Sens., vol. 54, no. 2, pp. 968-980, Feb. 2016.

[5] A. M. Zoubir, V. Koivunen, Y. Chakhchoukh, and M. Muma, "Robust Estimation in Signal Processing: A Tutorial-Style Treatment of Fundamental Concepts," IEEE Signal Process. Mag., vol. 29, no. 4, pp. 61-80, Jul. 2012.

[6] E. Ollila and V. Koivunen, "Influence functions for array covariance matrix estimators," in Statistical Signal Processing, 2003 IEEE Workshop on, 2003, pp. 462-465.

[7] C. Croux, E. Ollila, and H. Oja, "Sign and rank covariance matrices: statistical properties and application to principal components analysis," in Statistical data analysis based on the L1-norm and related methods, Springer, 2002, pp. 257-269.

[8] S. Visuri, V. Koivunen, and H. Oja, "Sign and rank covariance matrices," J. Stat. Plan. Inference, vol. 91, no. 2, pp. 557-575, 2000. 